Conclusion Birthweight has a U-shaped relationship with obesity in middle age. This relationship tends towards being J-shaped after adjustment for adult height.

\section{IS THE RECENT RISE IN TYPE 2 DIABETES MELLITUS INCIDENCE FROM 1984 TO 2007 EXPLAINED BY THE TREND IN INCREASING BODY MASS INDEX? EVIDENCE FROM A PROSPECTIVE STUDY OF BRITISH MEN}

doi:10.1136/jech.2010.120956.47

${ }^{1} \mathrm{~S}$ L Hardoon, ${ }^{1} \mathrm{R}$ W Morris, ' $\mathrm{M}$ C Thomas, ' $\mathrm{S}$ G Wannamethee, ' $\mathrm{L}$ T Lennon, ${ }^{2} \mathrm{P} \mathrm{H}$ Whincup. ${ }^{1}$ Department of Primary Care and Population Health, Division of Population Health, UCL, London, UK; ${ }^{2}$ Division of Community Health Sciences, St George's, University of London, London, UK

Objective Incidence of type 2 diabetes mellitus (T2DM) in the UK has increased by two-thirds in the last decade. Understanding the reasons for the unfavourable trend may help inform efforts to curb future increases. The association between T2DM and adiposity has been established. However, few attempts have been made to quantify the contribution of adiposity changes to the observed time trend in T2DM. We estimated the proportion of the time trend in T2DM incidence in British men over 24 years that may be explained by increasing population body mass index (BMI) levels.

Design Longitudinal study.

Setting 24 British towns.

Participants 6460 men from a socially and geographically representative cohort of older British men, followed up for doctor-diagnosed T2DM incidence between 1984 (aged 45 to 65 years) and 2007 (aged 67 to 89 years) inclusive. Men with a diagnosis of diabetes before 1984 were excluded.

Main outcome measures The age-adjusted increase in the hazard of T2DM between 1984 and 2007 and the contribution of changing BMI to this increase, derived from Cox proportional hazards modelling with time-updated covariates.

Results Between the periods 1984-1992 and 1999-2007, the ageadjusted hazard of T2DM more than doubled (hazard ratio 2.33, $95 \%$ CI 1.75 to 3.10 ). An estimated $26 \%$ (95\% CI 17 to 38 ) of this hazard increase could be statistically explained by a populationaveraged age-adjusted increase in BMI from 1984 to 1999 of $1.42 \mathrm{~kg} /$ $\mathrm{m}^{2}$ (95\% CI 1.10 to 1.74). Earlier and later portions of the follow-up were examined separately. Between 1984-1992 and 1992-1999, T2DM incidence increased by half (hazard ratio $1.59,95 \%$ CI 1.23 to 2.05). $22 \%$ (95\% CI 12 to 48) of this increase could be explained by rising BMI. Between 1992-1999 and 1999-2007, a similar increase in T2DM incidence was observed (hazard ratio $1.47,95 \%$ CI 1.17 to 1.84). $31 \%$ (95\% CI 17 to 81 ) of this increase was explained by BMI. Conclusion BMI changes can account for an appreciable portion of the increase in T2DM. Control and reversal of rising adiposity levels is therefore an important priority in controlling the diabetes epidemic. There remained a large "unexplained" portion of the T2DM increase. This may reflect imprecision of BMI as a measure of visceral fat or the independent contributions of changes in other determinants, such as physical activity and diet. The presence of other contributing factors would suggest the need for a more multifactorial approach to combat rising T2DM in the population.

\section{CHANGES IN WAIST CIRCUMFERENCE AND BMI IN ADOLESCENTS IN ENGLAND FROM 1977/1987 TO 2005-2007}

doi:10.1136/jech.2010.120956.48

1J Mindell, ${ }^{2} \mathrm{H}$ Dinsdale, ${ }^{2} \mathrm{C}$ Ridler. ${ }^{1}$ Health and Social Surveys Research Group, Department of Epidemiology and Public Health, UCL, London, UK; ${ }^{2}$ National Obesity Observatory, Oxford, UK

Objective To compare changes in BMI and waist circumference in the past 30 years among English children aged 11-16 years.
Design Cross-sectional health examination surveys.

Setting The Health Survey for England (HSE) is a survey of a random sample of the general population living in private households in England.

Participants A new, nationally representative sample is selected each year. 1988 children aged 11-16 years had a nurse visit in the three HSE years 2005-2007, of whom 1770 had valid waist circumference measurements. HSE data were compared with age-specific centile charts for waist circumference for British children aged 5.0-16.9 years derived from baseline surveys of 3585 boys in 1977 and 4770 girls in 1987.

Main outcome measures BMI and waist circumference z-scores derived from the baseline data, as BMI and waist circumference vary by age and sex.

Results The mean $z$-scores for waist circumference for children aged 11-16 years in 2005-2007 was substantially higher than the mean zscore for BMI, for both sexes: WC 1.0 (95\% CI 0.93 to 1.1), BMI 0.54 (0.44 to 0.63 ) for boys; WC 1.3 (1.2 to 1.4 ), BMI 0.48 (0.40 to 0.56 ) for girls (both $\mathrm{p}<0.001)$. There were no significant differences by sex in mean z-score for BMI, weight or height but the mean waist z-score for girls was significantly higher than that for boys $(p<0.001)$. There were no significant differences by age in waist circumference or BMI All children except for those in the lowest decile of waist circumference for girls had an increase in waist circumference of at least +1 SD since the 1977/87 baseline. BMI z-score increased across the top nine deciles of the BMI distribution by $0.4 \mathrm{SD}$ ( $2^{\text {nd }}$ to $4^{\text {th }}$ deciles) to $0.9 \mathrm{SD}$ (top decile). There were no significant differences by sex in the change over time except for the $10 \%$ of girls with the largest waist circumference for their age (1.6SD in girls vs $1.4 \mathrm{SD}$ in boys, $\mathrm{p}<0.01)$. Conclusion Waist circumference in adolescents has increased more than BMI, which may result in greater obesity-related adverse health impacts in the future. The increase in waist circumference has been greater for girls than for boys. Increases in the mean z-score across the majority of the population suggests that the whole population is becoming more obese, although this effect is more pronounced at the upper end of the distribution.

\section{THE REVERSAL OF THE SOCIAL GRADIENT OF OBESITY AMONG WOMEN IN EGYPT: AN ANALYSIS OF TRENDS USING MULTIPLE CROSS SECTIONAL SURVEYS 1995-2008}

doi:10.1136/jech.2010.120956.49

${ }^{1,2} \mathrm{~A}$ Aitsi-Selmi, ${ }^{2} \mathrm{M}$ Marmot. ${ }^{1}$ Wellcome Trust Doctoral Fellow, London, UK; ${ }^{2}$ Department of Epidemiology and Public Health, University College London, London, UK

Background The global obesity epidemic is spreading rapidly with a social distribution that varies according to the level of economic development: as countries develop, the burden of obesity appears to shift from the rich to the poor. Studying these changes as they occur can help shed further light on the social processes that fuel the obesity epidemic and determine its social distribution. Egypt provides a case in point for this research. Findings would be relevant to other low-and-middle income countries but may also be generalisable to an extent to poor communities in high income countries. Objectives To examine the social distribution of obesity among Egyptian women by socio-economic status and how it has changed over time.

Design and methods Retrospective analysis using four nationally representative cross-sectional surveys (Demographic and Health Surveys) conducted in Egypt between 1995 and 2008. Socioeconomic status was defined as the highest reported educational level attained.

Setting Egypt.

Participants 64605 women between 15 and 49 years excluding pregnant women.

Main outcome measure Obesity: defined as BMI (height/weight ${ }^{2}$ ) equal to or above 30 . 